\title{
Máquina de aprendizagem como ferramenta de auxílio na análise comportamental no ensino da leitura
}

\author{
Gilberto Nerino de Souza Junior - Instituto de Ciências Exatas e Naturais - \\ Universidade Federal do Pará (UFPA) - gilbertonerinojr@gmail.com
}

Dionne Cavalcante Monteiro - Instituto de Ciências Exatas e Naturais - Universidade Federal do Pará (UFPA) - dionnecm@gmail.com

Adalberto B. C. Pereira - Instituto de Ciências Exatas e Naturais - Universidade Federal do Pará (UFPA) - adalbertobosco@gmail.com

Ellton Sales Barros - Instituto de Ciências Exatas e Naturais - Universidade Federal do Pará (UFPA) - elltonsalesbarros@gmail.com

Leonardo Brandão Marques - Laboratório de Estudos do Comportamento Humano Universidade Federal de São Carlos (UFSCar) - leobmarques@ gmail.com

Resumo. Este artigo descreve um modelo de máquina de aprendizado com o objetivo de auxiliar na análise comportamental de alunos com déficit de aprendizado no processo de ensino da leitura baseado em definições de dificuldade formuladas. Com essa análise o analista do comportamento poderá obter informações do aprendizado individualizado de alunos nas palavras do programa de ensino utilizado, que indicam o estado de conhecimento do aluno sobre uma determinada palavra.

Palavras-Chaves: leitura, aprendizagem, máquina de aprendizado, tarefas de ensino.

\section{Machine learning as a tool to assist in behavioral analysis to teach reading}

Abstract. This article describes a model of machine learning to assist in the analysis of behavior of students with deficit in learning in the teaching of reading based on difficulty settings made. With this analysis the behavior analyst can obtain information individualized from the learning of students in the words used of program of education to indicate the status of the student's knowledge about a particular word.

Keywords: reading, learning, machine learning, teaching tasks

\section{Introdução}

A análise comportamental, área ligada à psicologia experimental cognitiva (Claudio, 1982), historicamente estuda os processos da aprendizagem humana, possuindo técnicas e métodos capazes de facilitar e acelerar o processo de cognição humana, examinando questões como memória, atenção, percepção, raciocínio e criatividade.

Desde a década de 80 pesquisadores da área da psicologia cognitiva vem desenvolvendo e aprimorando no Brasil um programa de ensino que auxilia o processo de aprendizagem de leitura e escrita, com o objetivo de aprimorar o desempenho escolar de crianças que possuem dificuldades em ler e escrever, com ou sem histórico de fracasso (Souza et al, 2006). Um dos principais objetivos desse programa é sanar de maneira rápida e precoce problemas presentes em crianças que apresentam dificuldades 
no processo de alfabetização além de ajudar alunos que apresentam dificuldades com os procedimentos convencionais em sala de aula (Reis et al, 2009).

Segundo Azevedo (2001) a leitura e escrita são dois repertórios inicialmente distintos e que envolvem uma série de relações diferentes e ambos são tratados como tarefas distintas e complexas. Disso decorre que dificilmente um método de ensino, por melhor que seja, será igualmente eficiente para todos os alunos. Por isso, tem-se necessidade contínua de desenvolvimento de recursos instrucionais personalizados e eficientes para aqueles que têm mais dificuldades.

A meta do programa Aprendendo a Ler e Escrever em Pequenos Passos (ALEPP) é a aquisição das relações básicas entre as palavras impressas, as figuras que as representam e o nome falado das figuras. Averiguações experimentais para o ensino de leitura e escrita foram realizadas e deram origem a um programa individualizado para o ensino dessas habilidades (Rose et al, 1996) no qual vem sendo sistematicamente avaliado e aperfeiçoado (Souza et al, 2006). A versão original, aplicada com materiais convencionais, deu origem a uma versão computacional (Rosa et al, 1998), que garante aplicação uniformizada a todos os aprendizes e eficaz em estudos de laboratório (Souza et al, 2006).

Neste mesmo cenário foi desenvolvido um jogo chamado ALE-RPG, por Sarmanho et al (2011), que se utiliza das tarefas de um Gerenciador de Ensino Individualizado por Computador (GEIC) com o objetivo de adicionar fatores lúdicos e motivacionais que estimulassem as crianças a não abandonarem o programa de ensino. Contudo os estudos não se aplicaram para uma forma computacional inteligente que buscasse prever ou classificar o aprendizado dos alunos auxiliando no processo de análise do aprendizado.

Na última década muito se tem preocupado com a interação mais realista e natural entre os jogos digitais e o jogador. Novas pesquisas na área de Inteligência Artificial (IA) estão abrindo modelos e revelando padrões e novas características interessantes para a indústria de jogos (Nareyek, 2007).

Uma promissora subárea da disciplina de IA na área de jogos é o aprendizado de máquina, pois possibilitam, entre diversos métodos, o suporte à previsão e classificação computacional. Pode-se mencionar, por exemplo, o emprego do aprendizado de máquina em diversas áreas e em aplicações como: recomendação de produtos na internet, engenharia de automação de veículos e processamento de linguagem natural (Andrew, 2011).

Este trabalho tem por objetivo propor um modelo de máquina de aprendizado para ser utilizado em um jogo digital, a fim de analisar dados comportamentais do processo de aprendizagem da leitura. A máquina de aprendizado, neste trabalho, tem por objetivo avaliar o grau de aprendizagem da leitura em palavras individuais, utilizando para isto a análise do comportamento do aluno durante a sessão de ensino, buscando assegurar a garantia do aprendizado em uma palavra e a garantia de que o aluno não aprendeu a palavra por sorte ou exclusão em suas respostas.

\section{Trabalhos relacionados}

Existem diversos trabalhos relacionados com esse artigo além do ALEPP e do ALERPG. Alguns são destaques nessa sessão. 
A individualização das instruções é uma maneira efetiva considerada importante para a Inteligência Artificial na Educação (IAED), segundo Du Boulay (1998). O seu trabalho aborda a diferença educacional da IA em sistemas IAED comparado com sistemas de ensino convencionais usados em sala de aula ou métodos convencionas.

No artigo de Self (1998) há uma abordagem "buttom-up" para mostrar a relevância da inteligência artificial em sistemas de suporte ao ensino devendo ser primordialmente motivacional e pedagógico. Comenta que existem regras importantes para o Computer Based Learning (CBS), como: prover a individualização do ensino através de um modelo cognitivo e identificar as dificuldades inerentes do aprendizado.

A pesquisa desenvolvida por Mahlmann et al (2010) caracteriza a exploração das possibilidades de previsão de aspectos do comportamento do jogador e seu interesse no jogo com o aprendizado supervisionado para o titulo TombRaider: Underworld.

Já o trabalho de Puzenat et al (2010) mostrou que o processamento cognitivo humano pode ser avaliado utilizando uma rede neural adequada através de uma interface. Sua ideia foi, através de jogos digitais, mensurar ações do usuário com aprendizado de máquina. Em dois protótipos o autor detectou a idade mental e se uma criança é canhota ou destra.

\section{Programas de Ensino ALEPP e GEIC}

O programa de ensino denominado “ALEPP” (Rose et al, 1989), apresenta algumas características planejadas para ajudar pessoas com dificuldades em leitura e escrita. A primeira característica diz respeito ao fato do programa ser individualizado, o que permite que cada pessoa cumpra as atividades de ensino de acordo com seu próprio ritmo. Outra característica é a divisão dos repertórios a serem ensinados em pequenas unidades chamadas de tarefas de ensino. $\mathrm{O}$ aluno pode repetir as unidades de ensino caso não tenha alcançado o critério de aprendizagem previamente estabelecido, o que garante que o progresso ao longo do programa só ocorrerá depois da aquisição dos repertórios previamente ensinados, fazendo com que o programa seja um produto de um arranjo de contingências para promover a aprendizagem (Skinner, 1965).

O programa ensina relações entre palavras impressas, figuras e palavras ditadas por meio de um procedimento de emparelhamento com o modelo de referência. Neste procedimento, o aprendiz deve escolher entre duas ou mais alternativas, por exemplo, apontar uma figura entre outras duas ou apontar uma palavra impressa entre duas, indicando a alternativa equivalente com modelo. A organização da estrutura de ensino baseia-se nas pesquisas realizadas por Reis et al(2009) sobre responder relacionamentos para encadear os diferentes formatos de apresentação e relacionamento dos estímulos.

O GEIC (Orlando, 2009) é um sistema que viabiliza a autoria e a aplicação remota (à distância) de programas de ensino, e no qual se encontra programado a última versão do ALLEP. Além disso, possui componentes para gerenciamento de recursos humanos (especialistas, tutores e alunos) e para consulta e geração de relatórios.

A figura 1 ilustra algumas das tarefas que o GEIC pode implementar. Nas tentativas do tipo AC o modelo é falado e a escolha é um estímulo em formato de texto. Em tentativas do tipo BC o modelo é uma figura localizada na parte superior da tela e os estímulos de escolhas são apresentados em forma de texto. Já nas tentativas do tipo 
CB o estímulo modelo é um texto e os estímulos de escolhas são figuras (Marques et al, 2011).

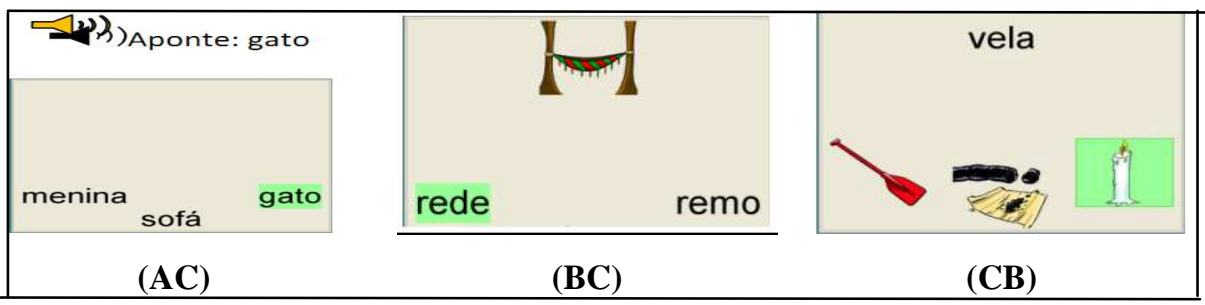

Figura 1 - Tipos de tentativas no GEIC.

\section{Modelo de Máquina de Aprendizado}

Esta sessão descreverá a máquina de aprendizado proposta onde está incluída a definição dos dados usados, a descrição da fórmula da dificuldade da tentativa para avaliar a dificuldade das tarefas de ensino mais adequadas ao aprendizado individualizado do aluno, e a técnica de classificação utilizada para avaliar o grau de aprendizagem das palavras.

\subsection{Definição dos dados}

O programa de ensino citado anteriormente possui diversos dados necessários que influenciarão na definição do aprendizado de máquina. Os dados coletados serão enviados para o modelo proposto a fim de iniciar o treinamento da IA e obter definições de padrões. Essas informações utilizadas para o aprendizado são:

- Acerto: Variável booleana que indicada acerto ou erro do aluno. Igual a 1 se o aluno acerta uma tentativa, caso contrário igual a 0.

- Estímulo Modelo: O estímulo modelo, ou simplesmente modelo, equivale à palavra que deve ser ensinada ao aluno na tarefa corrente. Toda tentativa ou tarefa tem um estímulo modelo que pode ser representado como figura, texto ou som.

- Estímulos Escolhas: Os estímulos escolhas são estímulos que serão colocados em uma tentativa de ensino para testar a capacidade de identificação em relação ao estímulo modelo. Podem-se ter diversas escolhas que o aluno poderá comparar com o modelo proposto.

- Número de escolhas: É o número de estímulos de escolhas de uma tentativa. Neste trabalho utilizou-se de 1 a 3 estímulos de escolhas incluindo o estímulo correto que é o estímulo que o aluno deve selecionar e está relacionado com o modelo.

- Tipo de Tentativa: Esse campo representa a relação de estabelecida entre os estímulos. Nele identificam-se os estímulos que são: som, figuras ou texto cujo foram utilizados nesse modelo os do tipo: AC, BC e $\mathbf{C B}$.

Esse trabalho focou na sessão de ensino do GEIC denominada de pré-teste, que tem o intuito de testar o aprendizado em 15 palavras: bolo, tatu, vaca, bico, mala, tubo, pipa, cavalo, apito, luva, tomate, vovô, muleta, fita e pato. 


\subsection{Fórmula da Dificuldade}

A fórmula da dificuldade proposta possui o intuito de definir um valor único de dificuldade por tarefa de ensino. O cálculo da dificuldade da tentativa agrega os diferentes fatores de dificuldade inerentes de uma tentativa em um dado numérico. Esses fatores de dificuldade foram definidos juntamente com especialistas da área de psicologia educacional de modo a obter um grau de complexidade da tarefa de ensino. Os fatores definidos foram:

- Proximidade das palavras: Para estabelecer uma métrica de dificuldade entre palavras semelhantes ou distintas, psicólogos especialistas na aquisição de leitura elaboraram uma tabela para classificar quanto uma palavra é semelhante à outra e com base nestes campos, avaliaram as palavras propostas cujo a média das aproximações foram formatadas na tabela 1, sendo que o grau de proximidade entre as palavras varia no intervalo $[0,1]$. Quanto maior o valor do grau de proximidade, maior a semelhança entre as palavras.

Tabela 1 - Proximidade das palavras. Amostra de 5 palavras.

\begin{tabular}{|c|c|c|c|c|c|}
\hline & tomate & vovô & muleta & fita & pato \\
\hline tomate & 1 & 0,225 & 0,35 & 0,275 & 0,5 \\
\hline vovô & 0,225 & 1 & 0,15 & 0,15 & 0,2 \\
\hline muleta & 0,325 & 0,15 & 1 & 0,525 & 0,25 \\
\hline fita & 0,275 & 0,15 & 0,525 & 1 & 0,375 \\
\hline pato & 0,5 & 0,2 & 0,275 & 0,55 & 1 \\
\hline
\end{tabular}

- Tipo de tentativa: para a relação inerente da dificuldade dos diferentes tipos de tentativas atribui-se um peso onde a soma desses pesos devem ser igual a $1 \mathrm{e}$ cada peso deve ser maior que zero. Logo os pesos foram definidos conforme a tabela a seguir.

Tabela 2 - Tabela com os pesos do tipo de tentativa

\begin{tabular}{|l|l|l|l|}
\hline Tipo de tentativa: & AC & BC & CB \\
\hline Peso: & 0,5 & 0,25 & 0,25 \\
\hline
\end{tabular}

- Número de escolhas: Em uma tentativa BC, por exemplo, o modelo será uma figura e as escolhas serão palavras escritas. Com isto, quanto maior o número de escolhas, maior a dificuldade de relação entre a escolha correta e o modelo.

Baseado nos atributos de dificuldade citados acima foi elaborada a equação 1 que concentra essas variáveis em um dado numérico:

$$
D= \begin{cases}\frac{E_{i=1}^{n-1} P_{i n}}{n-1} * \alpha+T * \beta+\frac{n}{n_{\max }} * \gamma & , n>1 \\ T * \beta+\frac{n}{n_{\max }} * \gamma & , n=1\end{cases}
$$

onde,

D: Dificuldade da tarefa,

Pin: fator de proximidade das escolhas incorretas em relação à palavra modelo, 
$T:$ peso da tentativa,

$n$ : número de escolhas.

$\boldsymbol{n}_{\max }:$ número máximo de estímulos de escolha. Neste trabalho considerou-se o máximo de 5 escolhas,

口, 口 e व: pesos para os fatores Proximidade das Palavras, Tipo de Tentativa, e Número de Escolhas, respectivamente, obtidos de forma empírica.

\subsection{Regressão Logística}

O método utilizado para realizar a previsão do aprendizado do aluno foi a regressão logística, que é utilizada para solucionar problemas de classificação de aprendizado supervisionado onde há uma variável de saída do tipo binário baseado em dados históricos de entrada (Medeiros et al, 2007). O objetivo de se usar a regressão logística é prever um grau de conhecimento para uma palavra específica, devendo ser executada para cada palavra modelo a fim de se conhecer um padrão de aprendizado do aluno para cada palavra.

A hipótese utilizada na regressão logística é mostrada na equação 2, que representará a fórmula de previsão e classificação dos dados que se deseja manipular:

$$
h\left(\theta^{T}, X\right)=\frac{1}{1+e^{-\theta^{T} X}}
$$

onde,

$X$ :matriz contendo os valores de dificuldade da tentativa,

ם: vetor com os coeficientes que o algoritmo deve determinar.

Para determinar os valores de $\mathbf{\square}$ foi utilizado o algoritmo de otimização chamado descida gradiente (gradient descent). Esse algoritmo é um método utilizado para encontrar o local mínimo da função através de passos contínuos segundo Thomas (2012) e Andrew (2011).

$\mathrm{Na}$ implementação desse trabalho foi integrado a regressão logística com o programa gerador da dificuldade das tentativas ilustrado na figura 2.

\begin{tabular}{|c|c|c|c|c|}
\hline proximidade de palavras.txt & $\begin{array}{l}\text { Programa Gerador } \\
\text { de Dificuldade }\end{array}$ & $\begin{array}{l}\text { Regressão } \\
\text { Logística }\end{array}$ & <<Gera $>>$ & $\begin{array}{l}\text { Previsão da } \\
\text { probabilidade do } \\
\text { acerto dado um valor } \\
\text { de dificuldade de uma } \\
\text { tentativa }\end{array}$ \\
\hline pesos dos tipos de tentativas.txt & Dificuldad & tativas.txt & & \\
\hline
\end{tabular}

Figura 2 - Diagrama do programa gerador das dificuldades com regressão logística.

\subsection{Decisão do aprendizado e parâmetros auxiliares}

Existem algumas condições e variáveis extras para a decisão do aprendizado de uma palavra proposta, que são:

- Condições Especiais (CondEsp): as condições especiais são um conjunto de condicionais que retornam true ou false podendo ser adicionadas novas condições conforme a necessidade do analista do comportamento. Essas condições especiais estão representadas na tabela a seguir. 
Tabela 3 - Quadro com as condições especiais.

\begin{tabular}{|c|l|l|}
\hline Condição & \multicolumn{1}{|c|}{ Descrição } & \multicolumn{1}{c|}{ Objetivo } \\
\hline $\mathrm{Q} \geq 7$ & $\begin{array}{l}\text { Quantidade de tentativas } \\
\text { realizadas (Q) }\end{array}$ & $\begin{array}{l}\text { Ter uma amostra de repostas da criança para executar o } \\
\text { treinamento da Machine Learning (ML). }\end{array}$ \\
\hline $\mathrm{E} \geq 1$. & $\begin{array}{l}\text { Pelo menos uma tentativa do } \\
\text { tipo E deve ser feita. }\end{array}$ & $\begin{array}{l}\text { O aluno deve montar uma palavra com as silabas ou } \\
\text { letras, demonstrando habilidade de escrita. }\end{array}$ \\
\hline
\end{tabular}

- Dificuldade Máxima (Dmax): é um valor máximo de dificuldade de uma tentativa para uma palavra modelo. Esse parâmetro é utilizado como limite de complexidade de uma tarefa de ensino para uma palavra específica. O sistema deve possuir uma dificuldade máxima para cada palavra.

- Média da Dificuldade Geral (Md): é um parâmetro estatístico que busca encontrar a média da dificuldade das tentativas para a palavra, mas somente para as tentativas com acerto. A condição para a média da dificuldade geral é $M_{d} \geq 50 \%$ da $D_{\max }$.

- Taxa de Acerto $\left(\mathbf{T}_{\mathrm{A}}\right)$ : equivale ao número de acertos dividido pelo total de tentativas executadas. Caso seja maior ou igual a $50 \%$ o seu valor é considerado true.

Objetivando encontrar um valor booleano de aprendizado da palavra treinada e preencher o campo sobre o conhecimento daquela palavra, este trabalho propõem a utilização da equação 3, onde a probabilidade de acerto é computada através da regressão logística mostrada na equação 4 e algoritmo 1:

$$
\text { CondEsp } \wedge M_{d} \wedge T_{A} \wedge \text { ProbA }=\text { True }
$$

onde,

ProbA: probabilidade de acerto gerada através dos métodos do programa da regressão logística conforme o algoritmo abaixo,

$$
\begin{aligned}
& \text { ProbA }=h_{\theta}\left(50 \% \text { da } D_{\max }\right) \\
& \text { Se ProbA }>0,5 \text { então, Indivíduo Conhece Palavra } \\
& \text { Senão, Indivíduo Não Conhece Palavra. }
\end{aligned}
$$

Com esse grau de conhecimento da palavra e a lógica da primeira ordem podemos obter dados mais próximos do real sobre o aprendizado do aluno na palavra treinada e testada. A figura 3 ilustra a máquina de aprendizado integrada com a lógica de primeira ordem apresentada acima. 


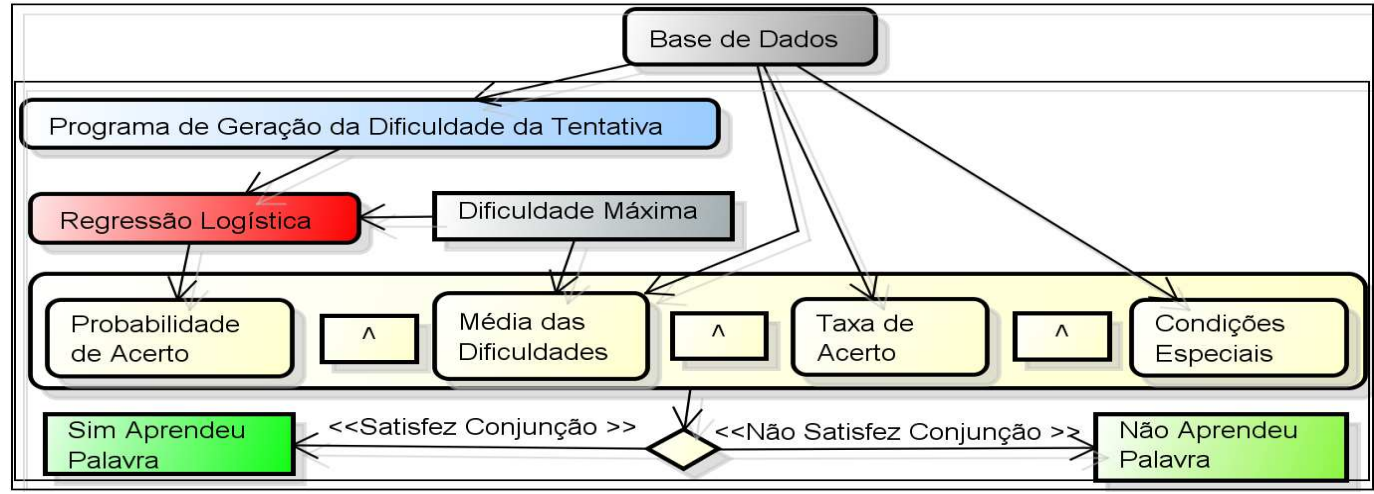

Figura 3 - Máquina de Aprendizado juntamente com a lógica de primeira ordem.

\section{Protótipo do modelo de máquina de aprendizado}

O protótipo da máquina de aprendizado desenvolvido para testes foi implementado na linguagem computacional Octave (OCTAVE, 2012).

O protótipo da ML foi construído baseado na estrutura apresentada na figura 4. Nessa estrutura nota-se que a base de dados possui transações de consulta, atualização e/ou inserção de dados. A atividade Execução do Pré-Teste Geral demonstra as primeiras apresentações de tentativas ao aluno, que já devem estar armazenadas na base de dados para que o conjunto de respostas seja registrado.

A atividade Lógica de Geração de Sessão é responsável por criar uma sessão para o aluno, ou seja, criar uma lista de palavras que o aluno ainda não aprendeu. Esta atividade está em conexão com a Base de Dados para identificar qual palavra deve ser adicionada na Lista de Palavras da Sessão de Aprendizado.

A Máquina de Aprendizado tem como entrada a lista de palavras de aprendizado, onde cada palavra da lista passará pelo aprendizado de máquina a fim de enviar, para a base de dados, os resultados do grau de conhecimento da palavra e se o aluno aprendeu ou não a palavra.

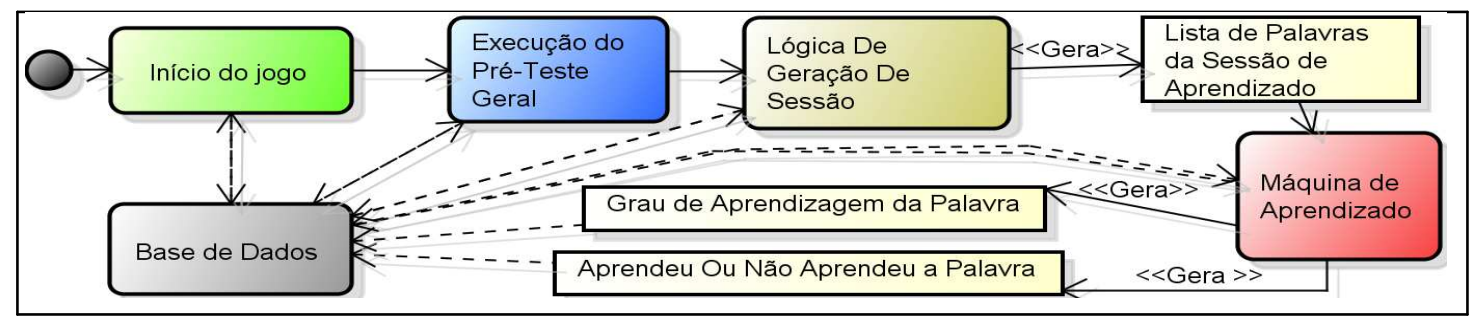

Figura 4 - Estrutura do protótipo da máquina de aprendizado.

\section{Resultados}

Os primeiros testes foram efetuados com base na análise de relatórios de desempenho no ALLEP executados por meio do GEIC. Esses relatórios representam as sessões de ensinos já realizadas por crianças no programa de ensino no qual se buscou avaliar o protótipo além de gerar alguns resultados preliminares.

A palavra escolhida na simulação foi a palavra "muleta" em que a tentativa de dificuldade máxima $\left(D_{\max }\right)$ é igual a $\mathbf{0 , 7 3 5 9 4}$. Foram induzidos 8 erros e 10 acertos 
nas tentativas. Duas simulações foram realizadas com os mesmos dados de treino para ambos, com sequências de erros e acertos diferentes. Os resultados da dificuldade da tentativa são mostrados na figura a seguir.

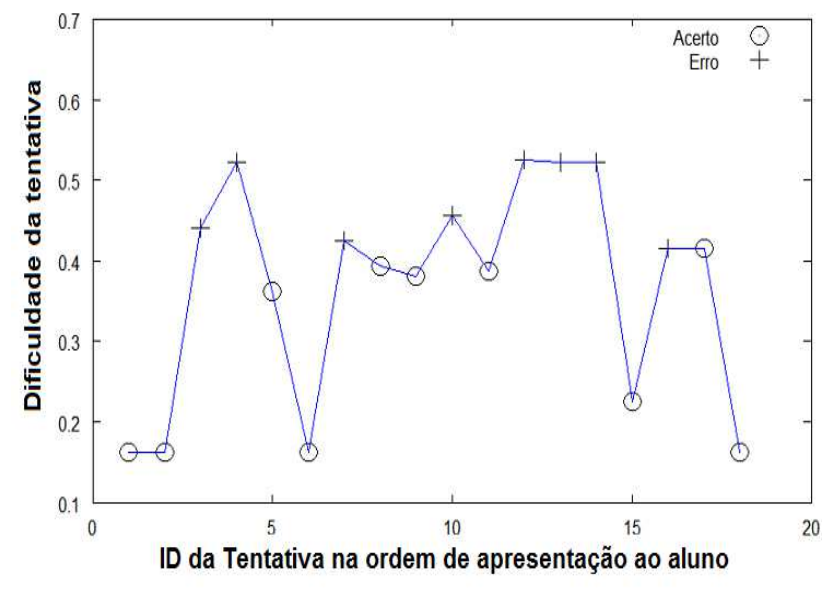

(a)

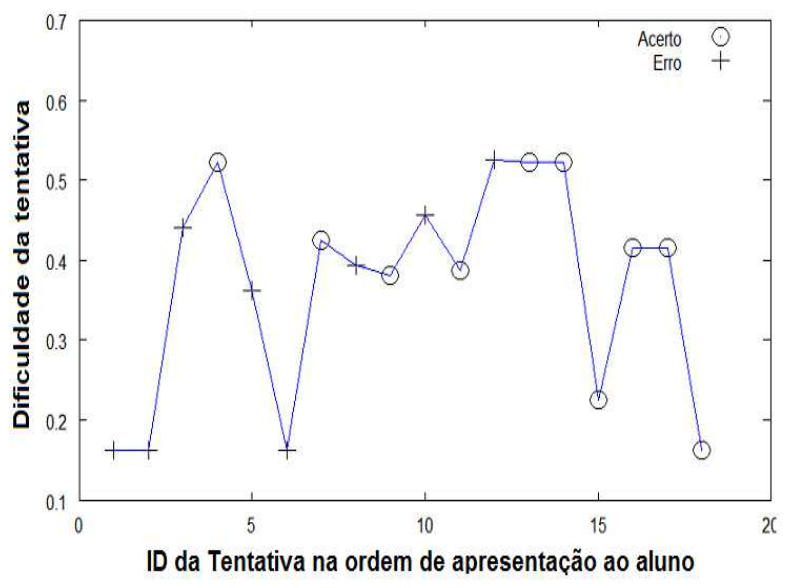

(b)

Figura 5 - Gráficos dos dados de treino do primeiro experimento (a) e do segundo experimento(b).

O primeiro experimento consiste em induzir erros para as tentativas que contêm maiores dificuldades. Desta forma busca-se simular possível déficit de aprendizado de um aluno que tem dificuldade de acertar tarefas complexas. Para os dados da figura 5(a) o programa gerou alguns dados, como consta na tabela 4, onde podemos observar que o indivíduo não aprendeu a palavra e o grau de aprendizagem adquirido na palavra é muito pequeno. Isso nos indica provável déficit de aprendizado. Classifica-se a palavra muleta como palavra que o aluno ainda não aprendeu.

O segundo experimento consiste em aplicar alguns erros iniciais e à medida que as tentativas são expostas, diminui-se o número de erros gradativamente até que por fim, no final da sessão, obtenham-se somente acertos independentemente da dificuldade da tentativa. Dessa forma busca-se simular o aprendizado gradativo do aluno. Para os dados da figura 5(b) o programa gerou algumas informações, onde a palavra muleta é classificada como palavra que o aluno aprendeu e obteve grau de aprendizagem de cerca de $83 \%$, conforme a tabela 4 .

Tabela 4 - Tabela dos resultados da primeira e da segunda simulação.

\begin{tabular}{|c|c|c|c|c|c|}
\hline Simulação & $\left(M_{d} \geq 50 \%\right.$ de $\left.D_{\text {max }}\right)$ & $\left(T_{A} \geq 0.5\right)$ & ProbA $>0,5$ & $\begin{array}{c}\text { Aprendeu } \\
\text { Palavra (AP) }\end{array}$ & $\begin{array}{c}\text { Grau do } \\
\text { Aprendizagem da } \\
\text { Palavra (GA) }\end{array}$ \\
\hline $\mathbf{1}^{\mathbf{a}}$ & $\begin{array}{c}\mathrm{M}_{\mathrm{d}}=0,28156 \\
\text { False }\end{array}$ & $\begin{array}{c}\mathrm{T}_{\mathrm{A}}=0,55556 \\
\text { True }\end{array}$ & $\begin{array}{c}\text { ProbA }=0,99880 \\
\text { True }\end{array}$ & False & $\mathbf{3 , 8 0 0 5 E - 2 1}$ \\
\hline $\mathbf{2}^{\mathbf{a}}$ & $\begin{array}{c}\mathrm{M}_{\mathrm{d}}=0,39781 \\
\text { True }\end{array}$ & $\begin{array}{c}\mathrm{T}_{\mathrm{A}}=0,55556 \\
\text { True }\end{array}$ & $\begin{array}{c}\text { ProbA=0,55653 } \\
\text { True }\end{array}$ & True & $\mathbf{0 , 8 3 9 5 4}$ \\
\hline
\end{tabular}

Para validação do trabalho utilizou-se um questionário expondo os gráficos da figura 5 a 3 especialistas da área da análise comportamental da UFSCar a fim de classificarem a evolução do aprendizado da palavra. Segundo a escala de concordância adotada (insuficiente $=1$, regular $=2$, bom $=3$, ótimo $=4$, excelente $=5$ ) foi realizada uma distribuição em percentual correspondente o que resultou nas informações da tabela 5, onde GA significa Grau de Aprendizagem e AP se houve Aprendizado na Palavra. 
Tabela 5 - Tabela dos resultados classificados pelos psicólogos para as simulações.

\begin{tabular}{|c|c|c|c|c|c|c|}
\hline & \multicolumn{2}{|c|}{ 1ㅇ Especialista } & \multicolumn{2}{|c|}{ 2o Especialista } & \multicolumn{2}{|c|}{ 3 Especialista } \\
\hline 1a Simule & $\mathbf{G A}=40 \%$ & $\mathbf{A P}=\mathrm{NÃO}$ & $\mathbf{G A}=40 \%$ & $\mathbf{A P}=\mathrm{NÃO}$ & $\mathbf{G A}=40 \%$ & $\mathbf{A P}=\mathrm{NÃO}$ \\
\hline 2a Simulação & $\mathbf{G A}=60 \%$ & $\mathbf{A P}=\mathrm{SIM}$ & $\mathbf{G A}=80 \%$ & $\mathbf{A P}=\mathrm{SIM}$ & $\mathbf{G A}=80 \%$ & $\mathbf{A P}=\mathrm{SIM}$ \\
\hline
\end{tabular}

Nota-se que os dados dos especialistas na área de análise comportamental estão de acordo com os dados de saída da máquina de aprendizagem, validando seus valores de saída.

\section{Considerações Finais}

Neste artigo foi apresentado um protótipo de máquina de aprendizado com simulações preliminares baseado na coleta de dados do programa de ensino GEIC para auxiliar o ensino da leitura às crianças com déficit de aprendizado. A simulação utilizada avaliou comportamentos mais frequentes do objeto de estudo desse trabalho.

A máquina de aprendizado apresentada permite a classificação do grau de aprendizado de palavras por aluno de forma adequada para o ensino da leitura em níveis iniciais, com o formato personalizado para cada aluno.

As futuras pesquisas oriundas deste trabalho se concentrarão em identificar padrões de comportamento para tipos específicos de tentativas e integração de geradores automáticos de tarefas em um jogo computacional, tendo como objetivo automatizar todo o ensino e aumentar a motivação do aluno.

\section{Referências}

ANDREW,N. G. Machine Learning Class. Disponível em: <https://d19vezwu8eufl6.cloudfront.net/ml/docs\%2Fslides\%2FLecture6.pdf $>$. Acesso em: 09set. 2011.

AZEVEDO, M. A.; MARQUES, M. L. Alfabetização hoje. São Paulo: Cortez, 2001.

CLAUDIO, J. T. Behaviorismo e Análise Experimental do Comportamento. In: Cadernos de Análise do Comportamento,3., p.10-23, 1982.

DU BOULAY, B. What does the "AI" in AIED buy? In: Artificial Intelligence in Educational Software. 1998.

MAHLMANN, T.; DRACHEN, A. ; TOGELIUS, J.; CANOSSA, A.; YANNAKAKIS, G. N. Predicting player behavior in Tomb Raider: Underworld. In: Computational Intelligence and Games, 14.,2010, p. 178-185,18-21.

MARQUES, L. B.; MEIO, R. G; MARIA, R. M. Manual do Usuário de Programas de Ensino via GEIC - Volume 1: Aprendendo a Ler e Escrever em Pequenos Passos. São Carlos, 2011.

MEDEIROS, S. A.; DANTAS, A.B.; DOS SANTOS, P. C. F.; MINUZZI, J. Modelagem com regressão logística para a satisfação no trabalho. In: IV Simpósio de Excelência em Gestão e Tecnologia (SEGET), 2007.

NAREYEK, A. Game AI is Dead. Long Live Game AI. In: Intelligent Systems, IEEE, vol.22, p.9-11. 2007.

OCTAVE. GnuOctave. Disponível em: 〈http://www.gnu.org/software/octave/>. Acesso em: 04 mai. 2012. 
ORLANDO, A.F.Uma Infra-Estrutura Computacional para o Gerenciamento de Programas de Ensino Individualizados, ed. São Carlos: UFSCar. 2009. Dissertação de Mestrado.

PUZENAT, D.; VERLUT I. Classification Behavior Analysis through Games Using Artificial Neural Networks. In: Advances in Computer-Human Interactions, p. 134-138. 2010.

REIS, T. S.; SOUZA, D. G.; ROSE, J. C. Avaliação de um programa para o ensino de leitura e escrita. In:Estudos em Avaliação Educacional, Caderno 20, p.425-50. 2009.

ROSA, A. B. Aprendendo a ler e escrever em pequenos passos. Documentação de Software para pesquisa. 1998.

ROSE, J. C.; SOUZA, D. G.; HANNA, E. S. Teaching reading and spelling: Exclusion and stimulus equivalence. In: Journal of Applied Behavior Analysis, p.451-69. 1996.

ROSE, J. C.; SOUZA, D. G.; ROSSITO, A. L.; ROSE, T. M. S. Aquisição de leitura após história de fracasso escolar: equivalência de estímulos e generalização. In: Psicologia: Teoria e Pesquisa, p.451-69. 1989.

SARMANHO, E. S.; SALES, E. B.; CAVALCANTE, D. M; MARQUES, L. B. Um Jogo com Reconhecedor de Voz para o Ensino de Crianças com Dificuldade de Aprendizagem em Leitura e Escrita. In: Semish, 2011.

SELF, J. Grounded in reality: the infiltration of AI into practical educational systems. In: Artificial Intelligence in Educational Software. 1998.

SKINNER, B. F. Tecnologia do ensino. In: Proceedings of the Royal Society. Londres: 1965.

SOUZA, D. G.; ROSE, J. C. C. Desenvolvendo programas individualizados para o ensino de leitura e escrita. In: Acta Comportamentalia, n. 14, p. 77-98. 2006

THOMAS, W. R. Redes Neurais Artificiais. Disponível em: 〈http://www.inf.ufes.br/ thomas>. Acesso em: 05mai. 2012. 